Preprint of http://dx.doi.org/10.1063/1.3295158

\title{
A SIMPLE LINE WAVE GENERATOR USING COMMERCIAL EXPLOSIVES
}

\author{
John S. Morris*, Scott I. Jackson* and Larry G. Hill* \\ ${ }^{*}$ Shock and Detonation Physics, DE-9, LANL, Los Alamos, NM 87545
}

\begin{abstract}
We present a simple and inexpensive explosive line wave generator which has been designed using commercial sheet explosive and plane wave lens concepts. The line wave generator is constructed using PETN- and RDX-based sheet explosive for the slow and fast components, respectively, and permits the creation of any desired line width. A series of experiments were performed on a 100-mm design, measuring the detonation arrival time at the output of the generator using a streak camera. An iterative technique was used to adjust the line wave generator's slow and fast components, so as to minimize the arrival time deviation. Preliminary tests achieved a wavefront simultaneity of $100 \mathrm{~ns}$ with a $7.0 \mathrm{~mm} / \mu$ s detonation wave. Designs, test results, and concepts for improvements are discussed.
\end{abstract}

Keywords: sheet explosives, line wave generator, plane wave lens, wave shaping, detasheet

PACS: $82.33 . \mathrm{Vx}$

\section{INTRODUCTION}

The explosive line wave generator (LWG) may be used to initiate slab charges along one edge or on irregularly shaped objects where simultaneous initiation along a given path is required. Similar designs have used sheet explosive and a variety of schemes to transform a single point initiation into the simultaneous arrival of a detonation wave along a line. Past designs have relied on modifications to the detonation pathway, making each path a predetermined length of explosive sheet to shape the output of the detonation. Using the methods shown in Fig. 1, care must be taken to ensure that the cross section of each channel is large enough to support detonation. Typically, the sheet explosive of choice is PETN-based detasheet with a composition of $63 \%$ PETN, $8 \%$ nitrocellulose, and $29 \%$ organic binder. The detasheet has a critical diameter greater than $0.3 \mathrm{~mm}$ [1].

Three common methods of creating a LWG using a single sheet are now discussed. The first of these methods uses a circular sector, Fig. 1a, of explosive sheet having a line width determined by the arc length of the terminal edge of the sector. The sector has apertures cut radially outward from the initiation point. To achieve the line wave output, the sheet is then deformed by creating a bulge in the center of the sector, allowing the terminal edge to be straightened into a line. As the detonation travels from the apex to the terminal edge of the sheet, the path length of each track is identical, producing a linear detonation wave reported to have an output simultaneity between 50 and $100 \mathrm{~ns}$ [2].

A second method of creating a line wave is to alter the detonation pathways through the use of holes cut into a triangular piece of sheet explosive, Fig. 1b, and thus creating equal path lengths to the terminal edge from the initiation point. The resulting output of this configuration is highly scalloped and only approximately a line if viewed several hole diameters downstream from the output edge.

The third method uses branching channels to generate a line output as shown in Fig. 1c. The channels are filled with an extrudable explosive and cut into a plastic backing plate such that all path lengths of the device are equal from the initiation point to the terminal end [3]. When using a sensitive explosive, the channels can be cut very thin to reduce the scallop- 


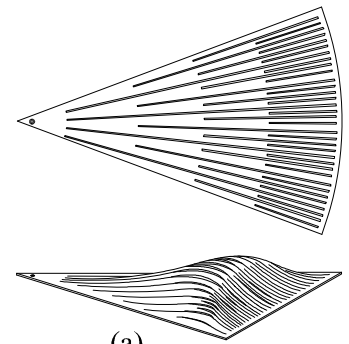

(a)

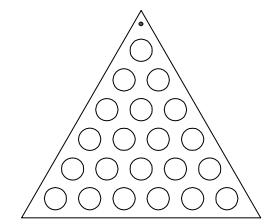

(b)

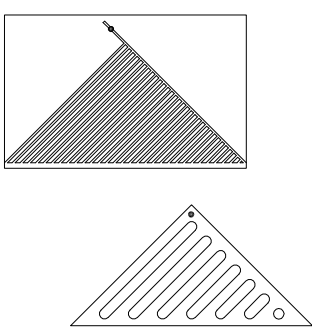

(c)

FIGURE 1. Three designs to produce line wave output: (a) circular sector, (b) perforated sheet, (c) branching method. The dot indicates initiation point for each design.

ing present in the LWG output. A typical output from the extruded branching-type LWG using XTX-8003 is shown in Figure 2 with an output simultaneity of $120 \mathrm{~ns}$.

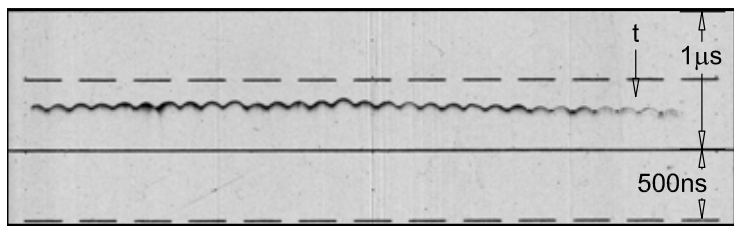

FIGURE 2. A streak image showing the arrival of the detonation front at the output end of a 150 -mm wide extruded branching-type LWG.

Similar devices have previously been fabricated using rigid, high-density explosives machined into the correct shape at a significant cost relative to the present design [4]. The benefit of our design is the reduction of scalloping in the output detonation and reduction in cost per device.

\section{THEORY OF DESIGN}

Our approach to creating a LWG is to take advantage of the difference in detonation velocities between two explosive sheet varieties: a $7.0-\mathrm{mm} / \mu \mathrm{s}$ "slow" PETN-based material and a 7.5-mm/ $\mu \mathrm{s}$ "fast" RDXbased material. The design is then based on plane wave lens theory: The transit time through any path on the lens must be identical and is equal to the sum of the transit time through the fast and slow components, with the centerline time serving as a reference.
From this concept, the simplest approach is to create the lens from an isosceles triangle of the slow sheet explosive with a base of the desired width, then line the outside edges of the triangle with the fast sheet material. The apex angle of this triangle is $\theta=2 \cos ^{-1}\left(D_{f} / D_{s}\right)$, where $D_{f}$ and $D_{s}$ are the fast and slow explosive component speeds, respectively [5]. A detonator located at the apex of the triangle acts as a point initiation source. The fast material initiates the slow material as the detonation front propagates through the height of the triangle and produces a line detonation at the terminal edge of the lens. Due to initiation issues of the RDX-based sheet material (discussed below), this simple method produced uncorrectable irregular output.

A more general approach is shown in Fig. 3, which represents the solution geometry for the solutions of the fast- and slow-component interface shapes. In order to determine the specific contour for each component, the user must select two characteristics of the desired lens: the half width of the lens $w$, and the detonator standoff distance $a$ from the slow-component. Large values of $a$ require increased accuracy when placing the detonator, while small values of $a$ increase the difficulty of cutting the apex section of the interface on both the slow and fast components.

Having selected the desired values for $a$ and $w$, the length of the lens is determined by equating the detonation transit time from the origin to the point $(l, w)$ on the centerline of the lens.

$$
\sqrt{l^{2}+w^{2}}=a+(l-a) \frac{D_{f}}{D_{s}}
$$

Making the substitutions of $\eta=D_{f} / D_{s}$ for the ratio of the detonation velocities, $\beta=\eta-1, \lambda=$ 


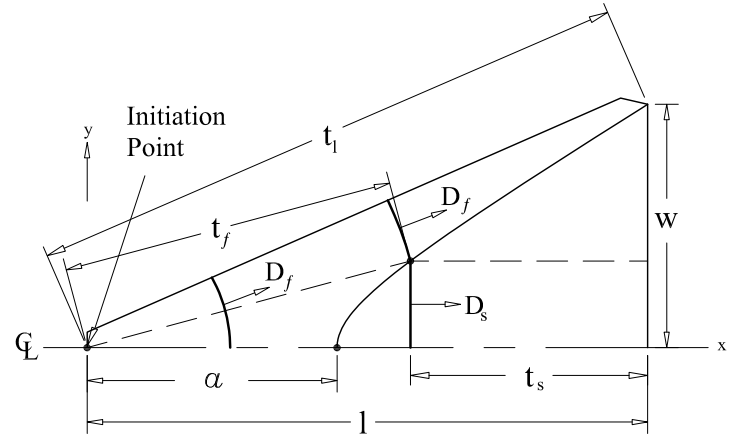

FIGURE 3. Geometry for a sheet explosive LWG. Origin located at the left with only the top half of the finished lens shown.

$\eta+1$ and solving for the length of the lens, Eq. 1 becomes

$$
l=\frac{a \beta \eta+\sqrt{\beta\left(a^{2} \beta+w^{2} \lambda\right)}}{\beta \lambda}
$$

where all values are known and a length can be calculated. The coordinates of the hyperbolic interface between the slow and fast explosive components are determined by using the same timing requirements as before, $t_{l}=t_{f}+t_{s}$, and solving for $y$ in Eq. 3 .

$$
\sqrt{l^{2}+w^{2}}=\sqrt{x^{2}+y^{2}}+(l-x) \eta
$$

The solution is shown in Eq. 4, with the substitution of $\zeta=\sqrt{l^{2}+w^{2}}$. The coordinates of the interface are obtained by evaluating $y$ from $a \leq x \leq l$.

$$
y=\sqrt{(\zeta-\eta(l-x))^{2}-x^{2}}
$$

\section{EXPERIMENTS}

It is clear from Eq. 4 that the detonation velocity ratio of the fast and slow explosive lens components is a critical parameter in the LWD design. Figure 4 illustrates the sensitivity of the lens contour and length to slight changes in detonation velocity ratio. Thus, it was important to accurately measure each of these velocities for the explosive tested.

All tests discussed herein used PETN-based Primasheet 1000 and RDX-based Primasheet 2000 sheet explosive, manufactured by Ensign-Bickford

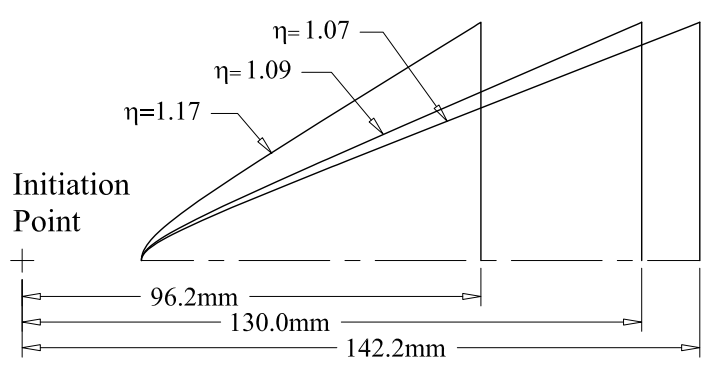

FIGURE 4. Detonation velocity ratio effect on LWG length.

Aerospace and Defense Company. The 2-mm explosive sheet thickness was used exclusively. Detonation velocities were measured by placing a $25-\mathrm{mm}$ wide by $300-\mathrm{mm}$ long strip of each explosive on a 4-mm thick PMMA backing plate. Nine ionization pins, spaced $25 \mathrm{~mm}$ apart, were located in the PMMA along the centerline of the test strip. The first pin was located $75 \mathrm{~mm}$ downstream of the initiation location. A series of three velocity measurements were recorded for each material averaged together. The averaged detonation velocities were found to be $7.002 \mathrm{~mm} / \mu$ s for the PETN-based explosive and $7.512 \mathrm{~mm} / \mu$ s for the RDX-based one.

With the measured velocities, values of $a=25 \mathrm{~mm}$ and $w=50 \mathrm{~mm}$ were selected and the resulting LWG was $96.2 \mathrm{~mm}$ in length. These parameters were used with Eq. 4 to generate the $(x, y)$ coordinates used to machine contour templates in PMMA for the LWG.

To shape the LWG components, the templates were placed onto the explosive and cut using a \#11 surgical blade. The cut pieces of sheet explosive were removed from the templates and aligned on a rectangular piece of 4-mm-thick PMMA. The initial detonator location was at the origin of the LWG, Fig. 3, with the output surface of the detonator parallel to the surface of the sheet explosive.

The RP-2 detonator used failed to initiate the RDX component, presumably because the run distance in the RDX-based sheet was greater than the thickness of the material, and the RP-2's output diameter was too small to sustain sufficient shock pressure to a depth of $2 \mathrm{~mm}$. The RP-2 detonator was replaced with a larger RP-80 detonator that was repositioned such that the detonator output surface was coincident with the origin shown in Fig. 3 and perpendicular to 
the LWG centerline. This modification successfully initiated the RDX-based sheet.

Several LWG's were tested and evaluated by imaging the time of arrival of the detonation front at the output surface using a Cordin 132 streak camera. Imaging light was generated by locating a layer of copper tape immediately downstream of the LWG output surface. This tape was covered with PETN paint, which was covered with a layer of clear PMMA. Arrival of the shock wave compressed and instantly ignited the paint, causing it to flash brightly through the PMMA and be recorded on the camera.

The results of three separate tests are shown in Fig. 5. Each streak record shows deviations from simultaneity of approximately $100 \mathrm{~ns}$. These vary from test to test and are attributed to user error in making each lens. We note that it is difficult to maintain proper blade angle when cutting around the apex contour and a $0.2-\mathrm{mm}$ error in cutting will produce a 30 -ns error in output.

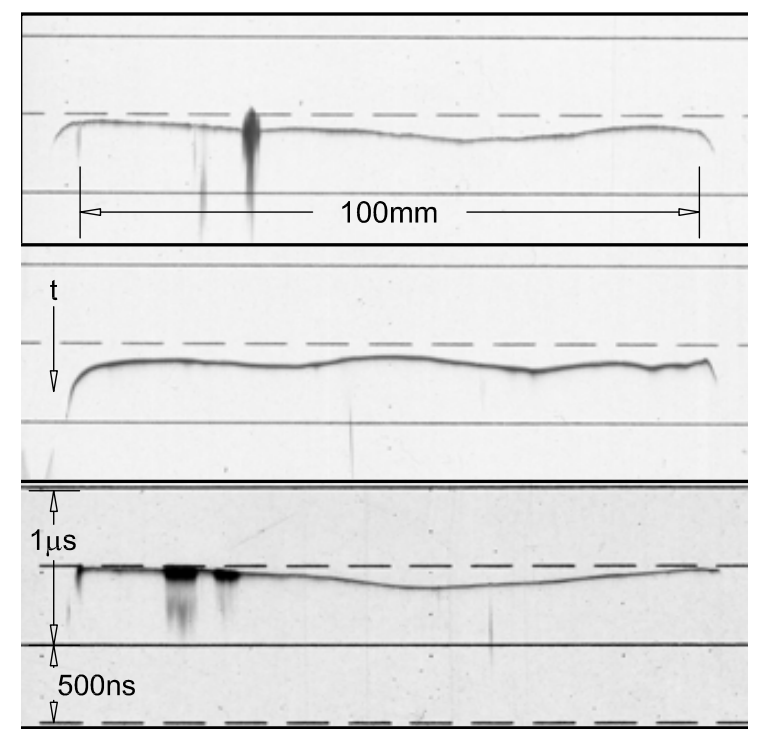

FIGURE 5. Streak images of the output of the 100-mmwide LWG design for three separate tests with an output simultaneity of $124 \mathrm{~ns}$ (top), $88 \mathrm{~ns}$ (middle), and $113 \mathrm{~ns}$ (bottom), respectively.

\section{FUTURE WORK}

As seen in the results from the hand crafted 100mm LWG, simply cutting the components from sheet stock using a razor blade introduces errors in arrival time which vary from shot to shot and person to person. However, depending on the required precision and the skill of the experimenter, this error may be acceptable.

In order to refine the templates used to create the LWG, hand-cutting operations should be removed from the operation. For the $100-\mathrm{mm}$ LWG, a set of cookie-cutter type dies can be made to replace the hand-cutting process and allow more precise control over the contour shape. Currently, the detonator has been treated as a point source which also introduces errors that can be further accounted for when the variability of hand fabrication is removed. These issues will be addressed in future work.

\section{CONCLUSIONS}

A simple and cost-effective line wave generator was designed using commercial sheet explosives with different detonation velocities. Preliminary hand-shaped tests achieved a wavefront simultaneity within $100 \mathrm{~ns}$ for a $7.0 \mathrm{~mm} / \mu$ s detonation wave. It is believed that future efforts can further increase the output simultaneity by replacing hand-shaping with a cookie-cutter type die.

\section{ACKNOWLEDGMENTS}

This work was funded by the Department of Energy. We thank Robert Mier and Larry Vaughan for their help in completing this project.

\section{REFERENCES}

1. Cooper, P. W., Explosive Engineering, WhileyVHC,Inc. New York, 1996.

2. Gibson, D., Explosive Line Wave Generator, US Patent 3,242,863 (1966), United States Atomic Energy Commission.

3. Coursen, D., Explosive Device, US Patent 2,943,571 (1960), E.I. duPont, Wilmington, DE.

4. Olinger, B., Personal Communication (2008).

5. W.C.Davis, "Introduction To Explosives," in Explosive Effects and Application, Springer-Verlag, New York, 1998, pp. 18-20. 